\title{
The Role of Carrier Transport on the Current Injection Efficiency of InGaAsN Quantum-Well Lasers
}

\author{
Jeng-Ya Yeh, Luke J. Mawst, and Nelson Tansu
}

\begin{abstract}
A theoretical and experimental study demonstrates that the current injection efficiency of InGaAsN quantum-well (QW) lasers can be significantly affected by carrier transport in the separate confinement heterostructure (SCH) region. An offset QW design is utilized to show the impact of hole transport on the temperature dependence of the external differential quantum efficiency and above threshold injection efficiency. A reduction of the current injection efficiency is found for structures which have significant hole transport times in the SCH.
\end{abstract}

Index Terms-Current injection efficiency, InGaAsN, long wavelength quantum-well $(\mathrm{QW})$ laser, material gain, temperature sensitivity, thermionic carrier leakage.

\section{INTRODUCTION}

$\mathbf{T}$ HE CURRENT injection efficiency $\eta_{\text {inj }}$ of quantum-well (QW) lasers, defined as the fraction of injected current or carriers which recombines (both radiatively and nonradiatively) in the QW, has a direct impact on both threshold current density and external differential quantum efficiency. Furthermore, the temperature dependence of $\eta_{\text {inj }}$ can be significant in some laser structures, leading to a high temperature sensitivity of both threshold current and external differential quantum efficiency. Improved understanding of the mechanisms impacting $\eta_{\text {inj }}$ provides a key to further optimize devices for efficient high output power and high temperature operation. Recent studies have indicated that $\eta_{\text {inj }}(T)$ plays a significant role in the observed strong temperature dependence of 1.3- $\mu \mathrm{m} \mathrm{InGaAsN}$ single QW lasers [1]. Here we report results of a theoretical and experimental study on the impact of carrier transport on $\eta_{\text {inj }}$ for InGaAsN QW lasers, providing insight into the design optimization of these laser structures.

In order to examine the effects of carrier transport on $\eta_{\mathrm{inj}}$, a laser structure design consisting of an asymmetrically placed QW was utilized. In this experiment, we focused on two laser structures for which the active region is intentionally placed close to either the $\mathrm{p}$-cladding or n-cladding layers. This method was also utilized by Nagarajan $e t$ al. to demonstrate the impact of QW position on device high-frequency modulation response performance [2]. With this design, we altered the scenario of carrier transport but fixed the threshold gain condition between these two lasers (i.e., same optical confinement factor $\Gamma$, mirror loss $\alpha_{m}$, and internal loss $\alpha_{i}$ ), resulting in nominally identical

Manuscript received December 6, 2004; revised February 28, 2005.

J.-Y. Yeh and L. J. Mawst are with the Reed Center for Photonics, Department of Electrical and Computer Engineering, University of Wisconsin-Madison, Madison, WI 53706-1691 USA (e-mail: jyeh@cae.wisc.edu).

N. Tansu is with the Center for Optical Technologies, Department of Electrical and Computer Engineering, Lehigh University, Bethlehem, PA 18015 USA.

Digital Object Identifier 10.1109/LPT.2005.852331 (a)

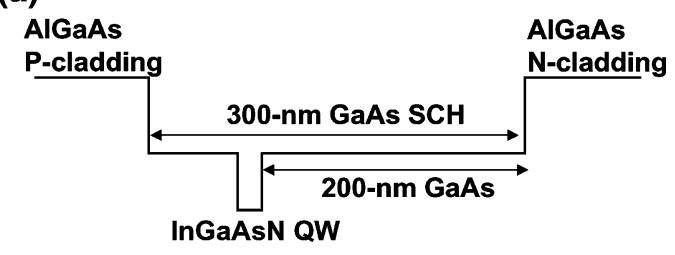

(b)

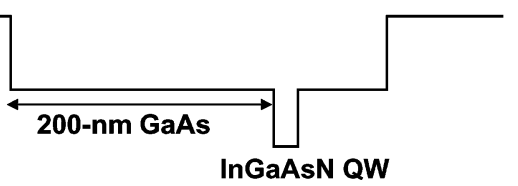

Fig. 1. Schematic band diagram of the offset $\mathrm{QW}$ structures. The active region is closer to (a) p-cladding for Laser P and (b) n-cladding for Laser $\mathrm{N}$.

values of threshold carrier density $N_{\text {th }}$. Therefore, identical recombination lifetime and thermionic escape lifetime of carriers in the QW are expected. This allows us to study directly the carrier transport effects on the device $\eta_{\text {inj }}$ without consideration of carrier recombination and leakage processes. The effect of carrier transport was analyzed using a theoretical model and good agreement with experiment was found.

\section{LASER Structure Design AND LASing Characteristics}

The laser materials were grown by low-pressure metal-organic vapor deposition with sources of trimethylgallium, trimethylaluminum, trimethylindium, $\mathrm{AsH}_{3}, \mathrm{PH}_{3}$, and dimethylhydrazine. The InGaAsN QW is located a distance $100 \mathrm{~nm}$ from the p-type cladding layer for Laser P, and $100 \mathrm{~nm}$ from the $\mathrm{n}$-type cladding layer for Laser $\mathrm{N}$ while keeping the thickness of the GaAs separate confinement heterostructure $(\mathrm{SCH}) 300 \mathrm{~nm}$ for both structures. Fig. 1 shows the schematic energy band diagrams of both Lasers $\mathrm{P}$ and $\mathrm{N}$. Not shown in Fig. 1 for simplicity, are 10-nm GaAs setback layers and 7.5-nm $\mathrm{GaAs}_{0.85} \mathrm{P}_{0.15}$ strain compensation layers surrounding the 8- $\mathrm{nm} \mathrm{In}_{0.4} \mathrm{Ga}_{0.6} \mathrm{As}_{0.995} \mathrm{~N}_{0.005} \mathrm{QW}$, as discussed in [3]. The 1.1- $\mu \mathrm{m}$-thick p-type and n-type AlGaAs cladding layers have a doping level of $1 \times 10^{18} \mathrm{~cm}^{-3}$ and the GaAs SCH region is undoped. The lasing characteristics were studied under pulse condition with a $1 \%$ duty cycle for broad area devices with $100-\mu \mathrm{m}$-wide stripes. The lasing wavelength is $1.29 \mu \mathrm{m}$ at room temperature. Fig. 2 shows the measured threshold current density $\left(J_{\text {th }}\right)$ and external differential quantum efficiency $\left(\eta_{d}\right)$ versus heat sink temperature for Lasers $\mathrm{P}$ and $\mathrm{N}$, both with 2-mm-long cavity lengths. At $20{ }^{\circ} \mathrm{C}, J_{\text {th }}$ is $249 \mathrm{~A} / \mathrm{cm}^{2}$ and $\eta_{d}$ is $31.7 \%$ for Laser $\mathrm{N}$, and improved to $207 \mathrm{~A} / \mathrm{cm}^{2}$ and $33.4 \%$ for Laser P. Both lasers share a similar $T_{0}\left(1 / T_{0}=1 / J_{\mathrm{th}} \times\left(d J_{\mathrm{th}} / d T\right)\right)$ value around $100 \mathrm{~K}$, but 


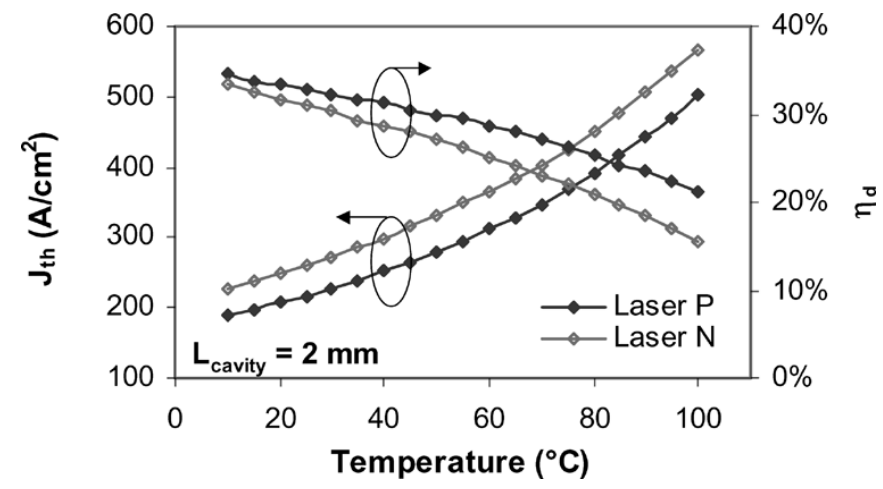

Fig. 2. Measured temperature dependence of threshold current density and external differential quantum efficiency of Lasers $\mathrm{P}$ and $\mathrm{N}$. Laser $\mathrm{P}$ has a smaller $J_{\text {th }}$ and higher $\eta_{d}$.

Laser $\mathrm{P}$ has an improved $T_{1}\left(1 / T_{1}=-1 / \eta_{d} \times\left(d \eta_{d} / d T\right)\right)$ value of $270 \mathrm{~K}$ compared to $175 \mathrm{~K}$ for Laser $\mathrm{N}$.

\section{CURRENT INJECTION EFFICIENCY MODEL AND EXPERIMENTAL RESULTS}

The current injection efficiency for InGaAsN QW lasers has been analyzed theoretically [4]. By using the steady-state two level rate equations considering carriers in the QW and barrier/SCH regions, the below-threshold $\left(\eta_{\text {inj_below_th }}\right)$ and above-threshold $\left(\eta_{\text {inj_above_th }}\right)$ current injection efficiency can be derived as follows [4]:

$$
\begin{aligned}
\eta_{\text {inj_below_th }}= & \frac{1}{1+\frac{\tau_{\text {bw }}}{\tau_{b}} \cdot\left(1+\frac{\tau_{\text {QW_total }}}{\tau_{\text {esc }}}\right)} \\
\eta_{\text {inj_above_th }}= & \eta_{\text {structure }} \\
& \cdot \frac{1-\Delta_{\mathrm{QW}} \cdot\left[\frac{1}{\eta_{\text {inj_below_th }}}-\beta \cdot\left(1+\frac{\tau_{\text {bw }}}{\tau_{b}}\right)\right]}{1+\frac{\tau_{b w}}{\tau_{b}}} .
\end{aligned}
$$

In these two equations, $\eta_{\text {inj_below_th }}$ and $\eta_{\text {inj_above_th }}$ are described by the lifetimes associated with the four dominant processes, including carrier radiative and nonradiative recombination in the $\mathrm{QW}\left(\tau_{\mathrm{QW}-\text { total }}\right)$ and $\mathrm{SCH}\left(\tau_{b}\right)$, ambipolar carrier transport in the $\mathrm{SCH}\left(\tau_{\mathrm{bw}}\right)$ [5], and thermionic leakage of carriers escaping out of the QW $\left(\tau_{\text {esc }}\right)$ [6]. In this model, the carrier capture time is not included due to its smaller value (around one order of magnitude) compared to the transport time $\tau_{\text {bw }}$ [7]. In (2), $\eta_{\text {structure }}, \Delta_{\mathrm{QW}}$, and $\beta$ represent the current injection efficiency from cladding region to $\mathrm{SCH}$ (i.e., structure dependent), the above threshold carrier unclamping rate, and spontaneous emission factor, respectively. In the ideal condition, $N_{\mathrm{QW}}$ remains pinned at and above laser threshold. In reality, $N_{\mathrm{QW}}$ may not be fully clamped and the carrier unclamping rate $\Delta_{\mathrm{QW}}$ is defined as the rate of increase of carriers in the QW due to an increase in the injection current, for the above threshold condition. It can be expressed as $\Delta\left(N_{\mathrm{QW}} \times V_{\mathrm{QW}} / \tau_{\mathrm{QW} \text { _total }}\right) / \Delta\left(I_{\mathrm{SCH}} / q\right)$, where $N_{\mathrm{QW}}, V_{\mathrm{QW}}$, and $I_{\mathrm{SCH}}$ are the carrier density in the $\mathrm{QW}$, volume of the $\mathrm{QW}$, and injection current from the cladding layers [4]. In this study, $\eta_{\text {structure }}$ and $\Delta_{\mathrm{QW}}$ were chosen to be $85 \%$ and $15 \%$, respectively, and assumed temperature independent. The less than unity $\eta_{\text {structure }}$ in the laser structure is typically resulted from the defect recombination at the poor interfaces in the cladding-SCH interface, leading to a reduction of the $\eta_{\text {structure }}$.
A careful examination for the carrier lifetimes of these four major processes is necessary. Details of $\tau_{\text {esc }}$ and the modeling method used here are extensively discussed in [1] and [4]. To evaluate $\tau_{\mathrm{QW} \text { _total }}$, a knowledge of the monomolecular, bimolecular, and Auger recombination coefficients $A, B$, and $C$, and their temperature dependence, are crucial. However, based on our calculation, we find that $\tau_{\mathrm{QW} \text { _total }}$ has little effect on the calculated $\eta_{\text {inj }}$ mainly due to a much higher temperature sensitivity of $\tau_{\text {esc }}$ for the structures under study. In our simulation, we utilized the published values of $A, B$, and $C$ parameters in [8]. The carrier transport behavior in the $\mathrm{SCH}$ obeys the ambipolar carrier transport and can be expressed by the equation as follows:

$$
\tau_{\mathrm{bw}}=\frac{1}{2} \cdot\left(\frac{L_{n}^{2}}{2 \cdot D_{n}}+\frac{L_{p}^{2}}{2 \cdot D_{p}}\right)
$$

where $L_{n}$ and $L_{p}$ are the thickness of GaAs SCH measured from the QW edge to n-cladding and p-cladding, respectively. The $D_{n}$ and $D_{p}$ are the diffusion coefficients of electrons and holes, respectively, which can be related to carrier mobility by the Einstein relation. Since the undoped GaAs SCH is background n-type $\left(n \sim 10^{16} \mathrm{~cm}^{-3}\right)$, the majority electron mobility and minority hole mobility were used in this calculation. Due to the significantly smaller value of hole mobility $\mu_{p}\left(\sim 400 \mathrm{~cm}^{2} / \mathrm{Vs}\right.$ at $300 \mathrm{~K})$ compared to electron mobility $\mu_{n}\left(\sim 9200 \mathrm{~cm}^{2} / \mathrm{Vs}\right)$ [9], $\tau_{\text {bw }}$ is ultimately determined by how fast holes can move across $\mathrm{SCH}$ region to $\mathrm{QW}$ and electron transport has a minimal effect. The significance of the temperature dependence of the minority hole mobility on $\eta_{\text {inj }}$ should also be noted. In our model, we utilized a $1 / T$ relationship for $\mu_{p}$, as observed by Lovejoy et al. [10], which results in a temperature-independent $D_{p}$. Therefore, $\tau_{\text {bw }}$ only slightly increases with temperature, which is attributed to $D_{n}$. Our calculations yield a smaller $\tau_{\mathrm{bw}}$ value of about $3-3.3 \times 10^{-12} \mathrm{~s}$ for Laser P compared to $9.82-9.88 \times 10^{-12} \mathrm{~s}$ for Laser $\mathrm{N}$, indicating the effect of the offset QW position. For calculating $\tau_{b}$, we utilized the following equations, considering carrier recombination in the $\mathrm{QW}$, thermionic escape rate, and carrier transport in the SCH [4]:

$$
\begin{aligned}
N_{B} & =N_{\mathrm{QW}} \cdot\left(\frac{1}{\tau_{\mathrm{QW} \_ \text {Total }}}+\frac{1}{\tau_{\mathrm{esc}}}\right) \cdot\left(\frac{V_{\mathrm{QW}}}{V_{B}}\right) \cdot \tau_{\mathrm{bw}} \\
\tau_{b} & =\frac{1}{A_{B}+B_{B} \cdot N_{B}}
\end{aligned}
$$

where $N_{B}, A_{B}, B_{B}$ are the carrier density, monomolecular, and bimolecular recombination coefficients in the $\mathrm{SCH}$ and $V_{B}$ is the volume of the SCH. The calculated $\tau_{b}$ of Lasers $\mathrm{P}$ and $\mathrm{N}$ both exhibit a monotonically decreasing behavior with temperature, reducing from $9.5 \times 10^{-9} \mathrm{~s}$ at $10^{\circ} \mathrm{C}$ to $8.2 \times 10^{-9} \mathrm{~s}$ at $100^{\circ} \mathrm{C}$ for Laser $\mathrm{P}$ and from $8.6 \times 10^{-9} \mathrm{~s}$ at $10^{\circ} \mathrm{C}$ to $6.1 \times 10^{-9} \mathrm{~s}$ at $100{ }^{\circ} \mathrm{C}$ for Laser $\mathrm{N}$. The longer $\tau_{b}$ for Laser $\mathrm{P}$ arises from its smaller $\tau_{\text {bw }}$ value compared to Laser N. Moreover, stronger carrier leakage at high temperature leads to an increased $N_{B}$ and, therefore, a reduced $\tau_{b}$.

To compare the experimental results with the model, $\eta_{\text {inj_above_th }}$ can be extracted experimentally by performing a conventional cavity length analysis [11]. Fig. 3 shows measured $\eta_{\text {inj_above_th }}$ as a function of temperature for the two laser structures, as well as the calculated values from the analysis 


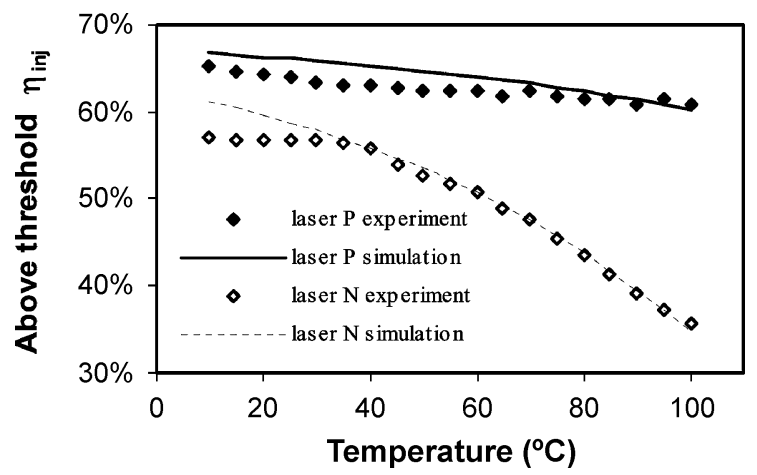

Fig. 3. Measured and calculated current injection efficiency above threshold as function of temperature.

(2). Useful insights can be obtained by measuring the temperature dependence of $\eta_{\text {inj_above_th }}$ and defining a characteristic temperature coefficient $\left(T_{\eta \text { inj_above_threshold }}\right)$ [12]. Shown in Fig. 3 are the measured characteristic temperature coefficient values of $\eta_{\text {inj_above_th }}$ (i.e., $T_{\eta \text { inj_above_threshold }}$ ), with values $1400 \mathrm{~K}$ and $190 \mathrm{~K}$ for Lasers P and N, respectively. With a shorter distance for holes to travel, Laser P not only exhibits an improved $\eta_{\text {inj_above_th }}$ compared with that of Laser N, but also a very high characteristic temperature, indicating low temperature sensitivity. The large difference of the temperature dependence of $\eta_{\text {inj_above_th }}$ for Lasers $\mathrm{P}$ and $\mathrm{N}$ can be attributed to the importance of the carrier transport behavior. Comparing the data shown in Fig. 3, although deviation in the lower temperature region occurs, this model accurately predicts the temperature dependence of the above threshold injection efficiency for the structures with an offset QW. Furthermore, the consistency between the experimental and calculated results validates this analytical model.

The high value of $T_{\eta \text { inj_above_threshold }}$ of Laser P does not necessarily lead to an equally high $T_{1}$ value (i.e., $T_{1}=175 \mathrm{~K}$ for Laser $\mathrm{N}$ and $270 \mathrm{~K}$ for Laser $\mathrm{P}$ ). This can be understood from the fact that, for Laser $\mathrm{P}$, the dominant factor influencing the $T_{1}$ value is not $T_{\eta \text { inj }}$ but $T_{\alpha i}$, the characteristic temperature coefficient of the internal loss $\alpha_{i}$. According to the temperature sensitivity analysis given in [12], $T_{1}$ can be expressed in terms of $T_{\eta \text { inj_above_threshold }}$ and $T_{\alpha i}$

$$
\frac{1}{T_{1}(L)}=\frac{1}{T_{\eta \text { inj_above_threshold }}}+\frac{\alpha i}{\alpha i+\alpha m(L)} \cdot \frac{1}{T_{\alpha i}}
$$

where the mirror loss $\alpha_{m}(L)=(1 / L) \times \ln (1 / R)$ is a function of cavity length $L$ and reflectivity $R$. Extracted from a temperature-dependent cavity length analysis, $T_{\alpha i}$ of Laser $\mathrm{P}$ is found to be only $189 \mathrm{~K}$. As a result, $T_{\alpha i}$ limits the temperature dependence of the laser efficiency (i.e., limits $T_{1}$ ) for Laser P, regard-

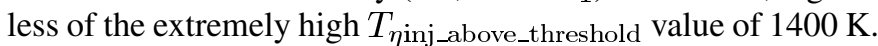
In addition, the much lower $J_{\text {th }}$ value observed for Laser P originates from the higher $\eta_{\text {inj_below_th }}$. From (1), the room temperature $\eta_{\text {inj_below_th }}$ and its characteristic temperature coefficient were calculated to be $86 \%$ and $300 \mathrm{~K}$ for Laser $\mathrm{P}$, and $64 \%$ and $120 \mathrm{~K}$ for Laser N. Thus, for Laser N, the $20 \%$ reduction of $\eta_{\text {inj_below_th }}$ and lower $g_{o J}$ compared to Laser P leads to the observed increase in $J_{\mathrm{th}}$.
It is also necessary to emphasize the crucial role of carrier leakage in this experiment. According to (1) and (2), the impact of carrier transport on $\eta_{\text {inj }}$ strongly depends on the competition between $\tau_{\mathrm{esc}}$ and $\tau_{\mathrm{bw}}$. For the InGaAsN QW structure, due to the process of hole thermionic escape, $\tau_{\mathrm{esc}}$ is estimated to be on the order of $10^{-12} \mathrm{~s}$. On the other hand, $\tau_{e}$ is estimated to be $10^{-11} \mathrm{~s}$ for an InGaAs QW with the same SCH structure [1]. Having a much longer $\tau_{\text {esc }}$, the impact of adjusting the InGaAs QW position to reduce the effective $\tau_{\mathrm{bw}}$ is not as significant as the case of the InGaAsN QW laser, and the model indicates that an almost indistinguishable $\eta_{\text {inj_above_th }}$ would result over a wide temperature range for an InGaAs QW. As a result, locating the QW close to p-type cladding layer does not necessarily lead to an improved $\eta_{\text {inj }}$, unless $\tau_{\text {esc }}$ is small enough so that the change of $\tau_{\mathrm{bw}}$ is relatively pronounced.

\section{CONCLUSION}

Two InGaAsN QW lasers with asymmetrically placed active region were carefully studied and compared. The experimental results were well predicted and explained by an analytical injection efficiency model. Improved $J_{\mathrm{th}}, \eta_{d}$, and $\eta_{\text {inj_above_th }}$ for the laser with the InGaAsN QW close to p-side indicates the importance of optimizing the QW location. The model allows for an improved understanding of the mechanisms of the carrier transport and injection efficiency of the diode laser, which is essential to achieving higher device performance.

\section{REFERENCES}

[1] N. Tansu and L. J. Mawst, "The role of hole leakage in 1300-nm InGaAsN quantum-well lasers," Appl. Phys. Lett., vol. 82, no. 10, pp. 1500-1502, Mar. 2003.

[2] R. Nagarajan, R. P. Mirin, T. E. Reynolds, and J. E. Bowers, "Experimental-evidence for hole transport limited intensity modulation response in quantum-well lasers," Electron. Lett., vol. 29, no. 19, pp. 1688-1690, Sep. 1993.

[3] N. Tansu, N. J. Kirsch, and L. J. Mawst, "Low-threshold-current-density 1300-nm dilute-nitride quantum well lasers," Appl. Phys. Lett., vol. 81, no. 14 , pp. $2523-2525$, Sep. 2002.

[4] N. Tansu and L. J. Mawst, "Current injection efficiency of InGaAsN quantum-well lasers," J. Appl. Phys., vol. 97, p. 054 502, Mar. 2005.

[5] R. Nagarajan, M. Ishikawa, T. Fukushima, R. S. Geels, and J. E. Bowers, "High-speed quantum-well lasers and carrier transport effects," IEEE J. Quantum Electron., vol. 28, no. 10, pp. 1990-2008, Oct. 1992.

[6] H. Schneider and K. V. Klitzing, "Thermionic emission and Gaussian transport of holes in a GaAs/AlGaAs multiple-quantum-well structure,"' Phys. Rev. B, vol. 38, no. 9, pp. 6160-6165, Sep. 15, 1988.

[7] H. Hirayama, J. Yoshida, Y. Miyake, and M. Asada, "Carrier capture time and its effects on the efficiency of quantum-well lasers," IEEE J. Quantum Electron., vol. 30, no. 1, pp. 54-62, Jan. 1994.

[8] R. Fehse, S. Tomic, A. R. Adams, S. J. Sweeney, E. P. O’Reilly, A. Andreev, and H. Riechert, "A quantitative study of radiative, Auger, and defect related recombination processes in 1.3-mu m GaInNAs-based quantum-well lasers," IEEE J. Sel. Topics Quantum Electron., vol. 8, no. 4, pp. 801-810, Jul. 2002.

[9] S. L. Chuang, Physics of Optoelectronic Devices. New York: Wiley, 1995.

[10] M. L. Lovejoy, M. R. Melloch, and M. S. Lundstrom, “Temperaturedependence of minority and majority carrier mobilities in degenerately doped gaas," Appl. Phys. Lett., vol. 67, no. 8, pp. 1101-1103, Aug. 1995.

[11] L. A. Coldren and S. W. Corzine, Diode Lasers and Photonic Integrated Circuits, 1st ed. New York: Wiley, 1995.

[12] N. Tansu, Y. L. Chang, T. Takeuchi, D. P. Bour, S. W. Corzine, M. R. T. Tan, and L. J. Mawst, "Temperature analysis and characteristics of highly strained InGaAs-GaAsP-GaAs (lambda $>1.17 \mu \mathrm{m}$ ) quantumwell lasers," IEEE J. Quantum Electron., vol. 38, no. 6, pp. 640-651, Jun. 2002. 